PROFESIONALES Y HERRAMIENTAS PARA EL DESARROLLO LOCAL Y SUS SINERGIAS TERRITORIALES. EVALUACIÓN Y PROPUESTAS DE FUTURO IX Coloquio Nacional de Desarrollo Local del GTDL-AGE 

ANTONIO MARTÍNEZ PUCHE, XAVIER AMAT MONTESINOS, ISABEL SANCHO CARBONELL y DANIEL SANCHIZ CASTAÑO (EDS.)

\section{PROFESIONALES Y HERRAMIENTAS PARA EL DESARROLLO LOCAL Y SUS SINERGIAS TERRITORIALES. EVALUACIÓN Y PROPUESTAS DE FUTURO}

IX Coloquio Nacional de Desarrollo Local del GTDL-AGE

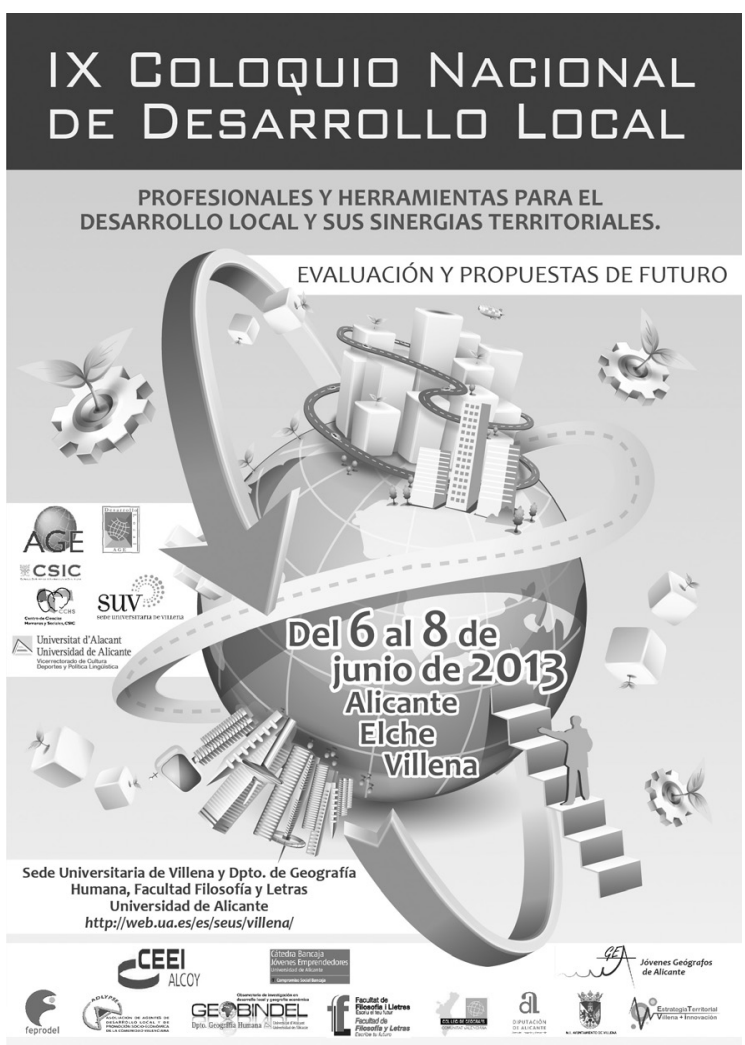


Este libro ha sido examinado y valorado por evaluadores ajenos a la Universidad de Alicante, con el fin de garantizar la calidad científica del mismo.

Publicacions de la Universitat d'Alacant

03690 Sant Vicent del Raspeig

Publicaciones@ua.es

http://publicaciones.ua.es

Telèfon: 965903480

(C) Antonio Martínez Puche, Xavier Amat Montesinos,

Isabel Sancho Carbonell y Daniel Sanchiz Castaño (eds.), 2016

(C) d'aquesta edició: Universitat d'Alacant

ISBN: 978-84-16724-00-0

Dipòsit legal: A 92-2016

Disseny de coberta: candela ink

Composició: Página Maestra (Miguel Ángel Sánchez Hernández)

Impressió i enquadernació: Guada Impresores

\section{unte \\ Unión de Editoriales
Universitarias Españolas \\ WWW.une.es
WWA}

Esta editorial es miembro de la UNE, cosa que garantiza la difusión y comercialización nacional y internacional de sus publicaciones.

Reservados todos los derechos. Cualquier forma de reproducción, distribución, comunicación pública o transformación de esta obra sólo puede ser realizada con la autorización de sus titulares, salvo excepción prevista por la ley. Diríjase a CEDRO (Centro Español de Derechos Repográficos, www.cedro.org) si necesita fotocopias o escanear algún fragmento de esta obra. 


\title{
EL OBSERVATORIO COMO HERRAMIENTA PARA EL DESARROLLO LOCAL
}

\author{
D. Teruel Roca \\ Universidad Autónoma de Barcelona \\ info@observatoriterresdelleida.gmail.com
}

\section{RESUMEN}

Los observatorios locales son una herramienta necesaria para el conocimiento de la realidad social y territorial de la localidad o el territorio donde se ubican. Permiten tener información detallada sobre indicadores demográficos, económicos y empresariales.

Una de sus finalidades es la creación de un foro de análisis y reflexión sobre la realidad socioeconómica y sus tendencias, promover el intercambio fluido de ideas, estudios y propuestas y elaborar informes sobre las líneas básicas de actuación de la política económica local-regional que pueden servir para la implantación de acciones de desarrollo local.

Palabras clave: observatorio urbano, Observatorios Sociales Locales, gestión pública.

\section{OBSERVATORY AS A TOOL FOR LOCAL DEVELOPMENT}

\section{ABSTRACT}

Local observatories are a necessary tool for understanding social and territorial reality of the village or territory where they are located. They allow to have detailed information on demographic, economic and business indicators.

One of its aims is to create a forum for analysis and reflection on the socio-economic situation and its trends, promote the fluid exchange of ideas, studies and proposals and reports on the basic guidelines of the local -regional economic policy as an implement of local development actions.

Keywords: urban observatory, Local Social Observatories, governance. 


\section{INTRODUCCIÓN}

El empeño de las ciudades más dinámicas por generar y ofrecer información socio-económica relevante y desagregada a nivel de zonas urbanas, tiene que ver su interés por prestar unos servicios más eficientes; pero también para desarrollar unas economías locales más competitivas.

En este contexto, el Observatorio Urbano de Palencia, responde a la voluntad del actual Consistorio por impulsar las nuevas tecnologías de la información, facilitando contenidos e información estadística, que incentiven su uso; para superar la brecha tecnológica que afecta a nuestras empresas y ciudadanos.

Difundir información sobre la estructura productiva de la ciudad, especialmente en lo que se refiere al comercio y los servicios de proximidad, para facilitar el análisis de la oferta existente. Comparar esos datos con la composición demográfica de sus residentes, para establecer la demanda potencial. Son análisis necesarios para elaborar escenarios que permitan evaluar el desarrollo de su actividad. Y todo ello, puede realizarse desde el Observatorio Urbano.

El Observatorio Urbano recoge información temática muy diversa relativa a la estructura demográfica, composición familiar, parque de vehículos, usos y fiscalidad del suelo, vivienda, aparcamientos y vados, IRPF, o actividad económica. -IAE-, o visitas turísticas. Así como toda una serie de datos que proceden de la explotación de registros administrativos, que solo puede ofrecer el Ayuntamiento, lo que les otorga un mayor interés.

Finalmente, el Observatorio Urbano es un instrumento que sirve para proyectar la ciudad en el mundo digital, para darle una mayor visibilidad. Posicionar la ciudad en la Web, ofreciendo contenidos actualizados, que permitan conocer con profundidad el marco socio-económico en el que se desarrolló, genera una imagen de ciudad mucho más atractiva y eficiente, ayudando de esta manera a que la marca de la Ciudad adquiera más valor, genera mayor confianza, al permitir que potenciales visitantes, estudiosos o inversores, puedan disponer de información actualizada desde una Web en la que se muestra al mundo la ciudad, trescientos sesenta y cinco días al año, veinticuatro horas al día.

Con el fin de cerrar la brecha entre las estadísticas locales y los diferentes actores locales, se propone la creación de Observatorios Sociales Locales, entendiendo que éstos son más que un sistema de información estadístico. Si bien la implementación de los Observatorios Locales es relativamente reciente, existen algunas experiencias que dan muestra de sus posibilidades y limitaciones. Una de estas experiencias es la de la Red de Observatorios Locales de la Diputación de Barcelona en España, la cual brinda en este trabajo un marco de referencia. 
Desde hace algunos años se aprecia el surgimiento de observatorios dedicados al análisis de diversos temas o problemas de la realidad social con igual diversidad de métodos, técnicas de recolección y análisis de datos. Así, se encuentran sobre temas de actualidad como, por ejemplo, la inmigración, la violencia familiar y la globalización, entre muchos. Otros, sobre temas más sensibles como es el caso de los derechos humanos y los tratados de paz; un ejemplo de estos últimos son los Observatorios de Derechos Humanos en las zonas metropolitanas, pueblos y áreas rurales de Brasil (Pinheiro, 2000) Aquellos, sobre aspectos y necesidades básicas, vale decir la Educación, la Salud, el Hábitat, el Desarrollo Humano, como es el caso del Observatorio para el Desarrollo Sostenible del Municipio de Manizales en Colombia (Velásquez, 2000). Los hay de carácter nacional, regional e internacional, públicos y privados. La lista de observatorios y sus tipos podría ser extensa pero de toda la variedad existente interesa, para este trabajo, destacar los observatorios orientados al estudio y reflexión de aspectos de la realidad social a escala local y, dentro de éstos, los que utilizan predominantemente indicadores sociales como herramientas centrales de trabajo.

\section{El ORIGEN DE LOS OBSERVATORIOS LOCALES}

El origen de los Observatorios Locales orientados a temas socioeconómicos se puede ubicar en Europa hacia finales de la década de los ochenta, aun cuando Desrosières (1996) afirma que fueron creados hacia la década de los sesenta en Francia por el INSEE (L'Institut National de la Statistique et des Etudes Economiques). Bien sea que hayan surgido en los ochenta o no, el hecho es que la instauración de estos espacios de análisis e interpretación de datos comienza su propagación por territorio europeo hacia comienzos de los noventa. Durante ese período, Soy (1991) destaca la aparición de varios Observatorios emblemáticos dentro de los cuales sobresalen el Observatorio de la Economía del Distrito de Poitiers en Francia, el Observatorio Económico-Territorial del Área Metropolitana de Milán, Italia, el del Sistema de Información Urbanística Regional de Madrid y, en Barcelona, el de Economía.

Todos estos observatorios poseen características comunes: 1) fueron promovidos por entes municipales y regionales a partir de la década de los ochenta; 2) tienen fines similares, «...los Observatorios constituyen un instrumento de recogida, producción y análisis de los datos y de información económica, social y territorial, con el objetivo de conocer la situación urbana y las transformaciones actuales y facilitar la toma de decisiones» (Soy, 1991:56); 3) tienen objetivos y metodologías específicas que giran en torno al tratamiento de los datos y de la integración de la información proveniente de las diversas fuentes existentes; 4) no sólo se limitan a disponer y utilizar los datos existentes, sino que tienen como función la interpretación de los hechos 
más significativos del desarrollo; 5) colocan un énfasis especial en los datos de carácter cualitativo así como en las áreas problemáticas o de especial interés, incluyendo la posibilidad de hacer encuestas o investigaciones ad hoc;6) integran métodos cualitativos y cuantitativos para el análisis e interpretación de datos. Crean indicadores específicos; 7) en términos organizativos, asignan importancia a su relación con los organismos productores de datos, tanto de carácter público como privado.

\section{LA RED DE OBSERVATORIOS LOCALES DE LA PROVINCIA DE BARCELONA}

Con la idea de profundizar en el estudio de los Observatorios Locales se presenta aquí la experiencia de los Observatorios Locales de Barcelona. La posibilidad de replicar o comparar esta experiencia en Venezuela hace necesario conocer el funcionamiento, organización, métodos y procesos de trabajo, así como los resultados alcanzados desde el momento de su creación hasta el presente. El análisis realizado de la experiencia de la Diputación de Barcelona se lleva a cabo mediante una investigación cualitativa, soportada sobre entrevistas focalizadas y observaciones semiestructuradas. También basado en informes, boletines, anuarios en papel y electrónicos proporcionados por los mismos observatorios, utilizando como marco muestral la Red de Observatorios.

Esta Red se articula a través de tres instancias: 1) la Diputación de Barcelona, la plataforma informática o servidor informático y los Observatorios. La Diputación tiene como su principal atribución la coordinación de los observatorios desde el Servicio de Fomento al Trabajo donde funciona física e institucionalmente la Red. 2) La plataforma informática es la unidad técnica encargada de efectuar la gerencia el Sistema de Información Socioeconómico Local (HERMES). Es también una aplicación informática de la red que reúne información socioeconómica de todos y cada uno de los municipios de la provincia de Barcelona. ${ }^{4}$ Mediante este servidor se agrupan todos los datos de carácter local indispensables para que los observatorios elaboren los indicadores, las tablas y los gráficos necesarios para los diferentes análisis. 3) Finalmente, los Observatorios Locales, son los elementos constitutivos de esa Red que agrupa a los 24 observatorios locales de Barcelona, entre los de Mercado de Trabajo y los de Actividad Económica.

A partir de la información proporcionada por la Diputación para este estudio, se seleccionan de manera opinática cinco observatorios sobre dos criterios: dependencia institucional y medios de difusión de la información.

- Dependiente del Consejo Comarcal y con difusión pasiva: Observatorio de Baix Llobregat.

- Empresa Municipal y con difusión pasiva: Observatorio Económico y Social de Terrassa. 
- Dependiente del Ayuntamiento y con difusión pasiva: Observatorio de Mercado de Trabajo de Mataró.

- Dependiente de Consejo Comarcal y con difusión activa: Observatorio de Trabajo y Economía de El Berguedà.

- Dependiente de un Consorcio y con difusión activa: Observatorio de Mercado de Trabajo de El Bages.

\section{FunCiONES DE LOS OBSERVATORIOS}

La necesidad de disponer de información actualizada de forma periódica por parte de los gobiernos locales define las funciones operativas de esta tipología de organismos e instituciones públicas:

- Recogida, sistematización, integración y actualización de la información disponible.

- Análisis de la información: descripción de la evolución socioeconómica.

- Generación de nuevas informaciones: estudios monográficos y ejecución de encuestas locales.

- Evaluación de escenarios y diagnosis territorial o sectorial.

- Propuesta de líneas estratégicas y acciones operativas.

- Comunicación, difusión y transferencia del conocimiento.

\section{CONCLUSIONES}

1. El observatorio debe representar siempre un apoyo útil a la toma de decisiones, especialmente en poner en marcha procesos operativos de análisisevaluación-propuesta y de transmisión ejecutiva de los resultados (a tiempo y en formato inteligible para quienes toman las decisiones).

Consecuentemente, los resultados deben ser útiles en tiempo útil. Los métodos que se implementen deben ser ágiles y más cualitativos. El trabajo del observatorio no únicamente se reduce a los reportes finales. Lo que importa es la asunción del cliente y el uso que haga de su contenido y de sus conclusiones. Es preciso hacer el conjunto útil y transparente para infundir confianza y capacidad de crítica (a pesar de resultados adversos de los análisis).

En consecuencia el observatorio debe ser fundamento esencial para construir un sistema de aprendizaje y innovación constante de la organización, esto es implantar una organización basada en el conocimiento.

2. La eficacia del sistema de observación se basa esencialmente en poder anticiparse a los hechos y a los efectos. Es, por tanto, tremendamente relevante el establecimiento de dispositivos altamente enfocados a ir más allá de las estadísticas, mediante el abordaje de recursos de información informales que permitan el establecimiento de sistemas de alerta y de vigilancia inteligentes. 
3. En esa misma línea, es especialmente relevante el trabajo con información cualitativa de primera mano, como valor añadido al análisis cuantitativo; a base de:

- Desarrollo de sistemas de información conceptuales y de vigilancia tecnológica.

- Detección, conocimiento e intensificación del acceso y inclusión en los procesos de los llamados grupos de expertos: think-tanks e, incluso, los local champions.

4. Investigación y desarrollo en el campo de la gestión eficaz y eficiente del conocimiento. A eso cabe explicitar que los observatorios deben ser por definición una organización enfocada a la valorización del conocimiento y, consecuentemente, al planteamiento de una organización muy enfocada a la innovación metodológica.

Teniendo en cuenta las posibilidades en cuanto a recursos escasos de personal y estructura, los observatorios deberán basar sus actividades de mejora y perfeccionamiento en:

- El despliegue de relaciones de cooperación, estrechas, fluidas e intensas con centros de investigación en el territorio en el campo del análisis territorial y la evaluación de políticas.

- La participación en redes profesionales de análisis socioeconómico, que promuevan el benchmarking territorial y entre organizaciones, y permitan una intensa actividad de intercambio de conocimientos aplicados y experiencias.

5. Finalmente, es importante señalar el rol de los observatorios en conjugar el análisis socioeconómico con la evaluación de las políticas públicas territoriales, de forma que se cierre el ciclo de la gestión pública dando conciencia que el impacto de las acciones implementadas no se valora únicamente por los resultados expost, sino por el impacto final, a medio plazo, sobre la calidad de vida de los ciudadanos. De ahí el papel esencial de los observatorios en el espíritu de la evaluación de las políticas públicas y, por tanto, en su necesaria renovación.

BiBLIOGRAFÍA

ACTIVA PROSPECT SL, Diagnosi dels observatoris locals del mercat de treball de la Diputació de Barcelona, Barcelona, Diputació de Barcelona, 2003.

DIPUTACIÓ DE BARCELONA, Hermes, web site: http://www.diba.es/hg2/ menu_pre.asp

DIPUTACIÓ DE BARCELONA, Hermes: Observar per actuar, Actas de las Jornadas, Barcelona, Pati Manning, 6-7 mayo, 2003, http://www.diba.es/promoeco/documents/hermes.asp 
OBSERVATORI ECONÒMIC I SOCIAL DE TERRASSA, Menú principal http://www.terrassa.org/laciutat/xifres/oest/oestindex.html

OBSERVATORI ECONÒMIC I SOCIAL DE TERRASSA, Terrassa en xifres, http://www.terrassa.org/laciutat/xifres/xifres.htm

SOY, Antoni, et URSA, Yolanda, Proposta per a l'endegament d'un observatori econòmico-social a la Ciutat de Terrassa, Barcelona, Centre d'Estudis de Planificació para el Ajuntament de Terrassa, 1991.

VARIOS ENTES MUNICIPALES, Proyecto El perfil de la Ciudad. Medida de la calidad de vida y la sostenibilidad de las ciudades medias europeas. http://www.terrassa.org/laciutat/xifres/estudis/perfildelaciutat/perfile.html 